\section{An exposure time effect in the Witkin rod-and-frame test}

\author{
R. W. HAYES and P. H. VENABLES \\ University of London, London, England
}

In the conventional Witkin rod-and-frame test (RFT), the effect of turning the rod with intermovement intervals of 1,4 , or $10 \mathrm{sec}$ was investigated. A highly significant difference was found between the errors made by the $26 \mathrm{Ss}$ in the three conditions. There was also a significant trend that indicated that increased exposure time impairs performance in the conventional RFT.

Witkin's (1954) rod-and-frame test (RFT) has been widely used by investigators in the area of personality and in the area of perceptual and motor skills, but a search of the literature reveals that one possibly important methodological variable of the test seems to have escaped attention. This is the duration of the interval between successive movements of the rod nearer to the vertical until the $\mathrm{S}$ says that the rod is upright. Karp (1963), for example, merely says that "Ss must instruct the experimenter to adjust the rod until it is vertical," while Witkin himself (1954) says, "The experimenter turns the rod in three degree steps, and the subject stops him when it appears straight to him." The majority of workers in fact simply fail to mention their procedure in this respect and are thereby ignoring the exposure time factor in the RFT.

There appear to be no previous studies of the effect of using different exposure times during the standard administration of the Witkin RFT with stepwise movements of the rod from the starting position towards vertical, and although two studies of the effect on RFT performance of differences of several minutes in exposure to the RFT display prior to the actual performance of the test have been reported, the findings are contradictory. Cohen \& Tepas (1958) found significant differences in error of tilt with differences of several minutes in prior exposure to the display before adjustment of the rod. Morant \& Aronoff (1966), using similar variations of prior exposure, failed to replicate the Cohen and Tepas finding. Morant and Aronoff suggest that the other authors' result cannot be explained, except as being due, perhaps, to the fading of the luminous paint used in the experiment. They say (in their own study, which used a rod and frame constructed from Lucite and Lumiline bulbs of constant though unspecified luminance) that the finding that the exposure time does not affect performance in the RFT accords with Koffka's (1935) principle that the main lines of organization provide the main directions of space against which the orientation of figures is judged and that the effect of these lines of organization does not increase over time or cause an aftereffect.

If Koffka's principle does hold in to the display, it would similarly be expected to apply to the normal Witkin RFT administration. On the other hand, if variation of exposure time during the standard Witkin RFT should prove to have a significant effect on performance, it would suggest that Koffka's principle does not apply to the RFT situation and that it may be that Morant \& Aronoff's (1966) rather than Cohen \& Tepas's (1958) findings are puzzling.

The present experiment investigates the effect of different exposure times to the display during the administration of the conventional Witkin RFT, modified only in using three different intermovement intervals as the rod is adjusted stepwise from its initial tilt toward upright, and the null hypothesis is that the increased exposure time will make no significant difference to performance. SUBJECTS

Ss were 26 naive female undergraduate or postgraduate students between 20 and 35 years old, chosen at random from 62 available Ss.

\section{APPARATUS}

Apparatus consisted of an experimental room, $6 \times 6 \mathrm{ft}$ and $71 / 2 \mathrm{ft}$ high, carpeted, light-proof, with a fanblower/extractor, intercommunication speaker/microphone, a metal-framed chair with a high back to support the head, and a luminous rod-and-frame apparatus of the same dimensions as that used by Witkin (1954). A modification of the original Witkin apparatus was that the axes of the tubes controlling the movement of the rod and of the frame passed through the rear wall of the experimental room so that the $\mathrm{E}$ was not sitting with the $\mathrm{S}$ and could read the protractor for degrees of error more easily. Both rod and frame could be tilted to an initial the RFT situation for prior exposure angle of $28 \mathrm{deg}$ from vertical, left or right, as in Witkin's RFT, and the brightness of the luminous rod and frame in darkness was made constant initially for all $\mathrm{Ss}$ by being given $5 \mathrm{~min}$ exposure to a 150-W lamp suspended at the level of the axis of the rod and 25 in. from the center prior to each set of 24 trials.

The 26 Ss were assigned randomly to testing sessions. On arrival, they were told that the object of the experiment was to investigate the relationship of certain personality variables to the ability of the individual to judge when a luminous rod viewed in complete darkness was upright. They were guided into the darkened experimental room with eyes closed and were seated with the head in an upright position facing the rod-and-frame display, which was already set to the starting position, as specified below. The $\mathrm{E}$ then left the room and continued instructions via the intercommunication system. Ss were asked to open their eyes to listen to the rest of the instructions. These instructions explained that the Ss were to keep their eyes closed until $\mathrm{E}$ said "Ready?" If ready, Ss were then to say "Yes" and open their eyes. The rod would then be moved in steps from the starting position towards vertical, and when Ss thought it was vertical, that is to say upright like a plumb line or a telegraph pole, they were to say "Now" and close their eyes until the next "Ready." The RFT trials were then begun, with the three exposure conditions of 1,4 , and

Table 1

Means of RFT Errors in Degrees

Intermovement Interval in Seconds

\begin{tabular}{rrrr}
\multicolumn{1}{r}{$s$} & 1 & 2 & \multicolumn{1}{c}{10} \\
\hline 3 & 1.00 & 2.25 & 2.50 \\
20 & 1.88 & 0.88 & 0.50 \\
5 & 2.00 & 3.13 & 9.38 \\
24 & 2.00 & 6.00 & 7.88 \\
4 & 2.13 & 3.88 & 3.88 \\
7 & 3.50 & 7.00 & 2.75 \\
17 & 3.63 & 8.13 & 4.13 \\
21 & 4.13 & 5.50 & 10.00 \\
14 & 4.50 & 7.38 & 9.75 \\
9 & 4.63 & 7.38 & 13.75 \\
10 & 5.00 & 9.13 & 9.75 \\
8 & 5.13 & 6.50 & 14.24 \\
26 & 5.38 & 6.75 & 9.38 \\
19 & 5.38 & 7.88 & 9.75 \\
16 & 6.50 & 9.75 & 12.00 \\
11 & 6.50 & 11.25 & 13.00 \\
18 & 9.13 & 12.50 & 16.13 \\
2 & 9.63 & 11.75 & 15.75 \\
1 & 9.75 & 13.75 & 17.50 \\
15 & 11.25 & 14.00 & 19.00 \\
22 & 13.13 & 16.13 & 20.63 \\
13 & 15.88 & 17.88 & 22.25 \\
23 & 18.63 & 22.50 & 23.50 \\
25 & 22.75 & 22.63 & 25.75 \\
6 & 23.13 & 23.75 & 26.00 \\
12 & 20.13 & 20.38 & 24.13 \\
\hline
\end{tabular}


$10 \mathrm{sec} / \mathrm{deg}$ being randomly allocated to the 24 trials to give eight trials in each condition. Each of the possible combinations of initial tilt $28 \mathrm{deg}$ left or right of vertical for rod and for frame was given an equal number of times for each exposure time condition.

RESULTS AND DISCUSSION

An analysis of variance of the results indicates a significant effect for the different exposure times $(\mathrm{F}=50.2770, \quad \mathrm{df}=2 / 50, \quad \mathrm{p}<.001)$, and Page's L test (Page, 1963) shows a significant trend $(\mathrm{L}=354.5, \mathrm{~m}=3$, $n=26, p<.01$ ), as would be expected from an inspection of the means, Table 1, and Fig. 1, which suggest an increase in error with increasing exposure times. The short $1-\mathrm{sec} / \mathrm{deg}$ exposure in the present experiment is that used for normal RFT work by the authors. The rearrangement of the means in Table 1 shows that in this short exposure condition, there are, by employing the criteria of less than 2-deg error for FI and more than 7-deg error for FD, 4 true FI, 10 FD, and 12 medium Ss. Inspection of Table 1 also shows that of $26 \mathrm{Ss}$, only 2 made more error in the short than in the long exposure, one of these, S 20, being FI and the other, S 7, a medium S.

$A$ second analysis of variance, grouping the 13 low- and 13 high-error $\mathrm{Ss}$, revealed that there was no significant Groups by Exposure Time Effects interaction ( $F=1.598$, df $=2 / 48, p>.05$ ).

In general, the results suggest that the null hypothesis is invalid, and that increased exposure time does increase error in the conventional Witkin RFT. We now have to interpret these results. Morant and Aranoff's suggestion, above, that fading of the luminous paint may increase error cannot explain the present findings as the 1-, $4-$, and 10-sec trials were in random order and not systematically related in time to initial activation. An

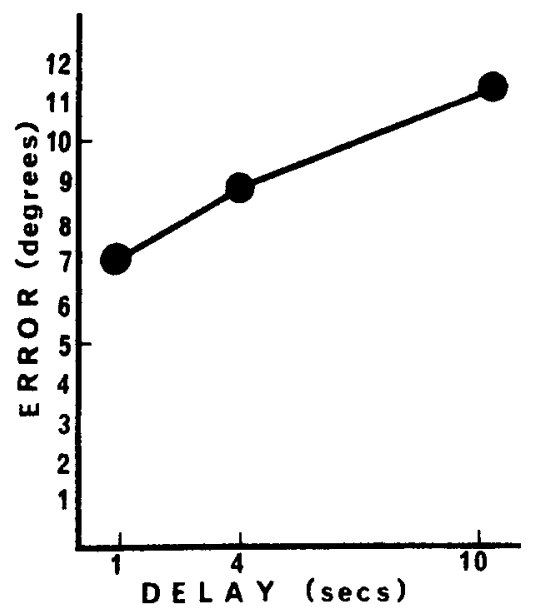

Fig. 1.

alternative explanation could be that in the shorter exposures the $S$, being in more of a hurry to make her decision, might have a higher level of arousal, which, on Oltmann's (1964) hypothesis, might give a narrowing of attention and thereby improve RFT performance. However, the effects of attempts to improve RFT performance by the use of "arousing stimuli" in Oltmann's original experiment were small and have not been found to be replicable (Vaught, 1969; Hayes \& Venables, 1970). Another possible explanation of the greater error in RFT with longer exposures is that it may relate to the adaptation to an optically rotated visual field as a function of length of exposure time demonstrated by Ebenholtz (1966). In the present experiment, the rotation is mechanical, the visual field in each trial being the tilted frame and the rod being the figure within the field. In this interpretation, with increasing adaptation as a result of increasing exposure to the tilted field, the rod would be expected to be set as subjectively "upright" at increasing deviations from the true vertical. This is in fact what happened. The advantage of this hypothesis is that, if it is supported by further research, it may allow some of the past work on the mechanism of adaptation to optical rotation of the visual field to be utilized in investigations of the basis of the individual differences in RFT performance, and it also suggests that the individual differences in adaptation to optical rotation which have been noted in earlier studies (Ebenholtz, 1966), but left without interpretation, may prove to be related to the established personality dimension of field dependenceindependence.

\section{REFERENCES}

COHEN, W., \& TEPAS, D. Temporal factors in the perception of verticality. American Journal of Psychology, 1958, 71, 760-763.

EBENHOLTZ, S. M. Adaptation to a rotated visual field as a function of degree of optical tilt and exposure time. Journal of Experimental Psychology, 1966, 72, 629-634.

HAYES, R. W., \& VENABLES, P. H. EEG measures of a rousal during RFT performance in "noise." Perceptual \& Motor Skills, 1970, 31, 594 .

KARP, S. A., POSTER, D. C., \& GOODMAN, A. Differentiation in alcoholic women. Journal of Personality, $1963,31,390$.

KOFFKA, K. Principles of Gestalt psychology. New York: Harcourt Brace, 1935.

MORANT, R. B., \& ARONOFF, J. Starting position, adaptation, and visual framework as influencing the perception of verticality. Journal of Experimental Psychology, 1966, 71, 684-686.

OLTMANN, P. K. Field dependence and arousal. Perceptual \& Motor Skills, 1964, $19,441$.

PAGE, E. B. Ordered hypotheses for multiple treatments. A significance test for linear ranks. Journal of the American Statistical Association, 1963, 58, 216-230.

VAUGHT, G. M. A reply to comments on "RFT stability or failure to arouse?" Perceptual \& Motor Skills, 1969, 29, 78. WITKIN, H., LEWIS, H. S., HERTZMAN, M., MACHOVER, K., MEISSNER, P. B., \& WAPNER, S. Personality through perception. New York: Harper, 1954. 